\title{
Electrical characterization of CdTe pixel detectors with Al Schottky anode
}

\author{
A.A. Turturici, L. Abbene*, G. Gerardi, F. Principato \\ Dipartimento di Fisica e Chimica,Università di Palermo,Viale delle Scienze, Edificio 18, Palermo 90128, Italy
}

\section{A R T I C L E I N F O}

\section{Article history:}

Received 16 April 2014

Received in revised form

3 July 2014

Accepted 3 July 2014

Available online 14 July 2014

\section{Keywords:}

CdTe

Schottky contacts

Polarization

Pixel detectors

$\mathrm{X}$-ray and gamma ray spectroscopy

\begin{abstract}
A B S T R A C T
Pixelated Schottky Al/p-CdTe/Pt detectors are very attractive devices for high-resolution X-ray spectroscopic imaging, even though they suffer from bias-induced time instability (polarization). In this work, we present the results of the electrical characterization of a $(4 \times 4)$ pixelated Schottky Al/p-CdTe/Pt detector. Current-voltage $(I-V)$ characteristics and current transients were investigated at different temperatures. The results show deep levels that play a dominant role in the charge transport mechanism. The conduction mechanism is dominated by the space charge limited current (SCLC) both under forward bias and at high reverse bias. Schottky barrier height of the $\mathrm{Al} / \mathrm{CdTe}$ contact was estimated by using the thermionic-field emission model at low reverse bias voltages. Activation energy of the deep levels was measured through the analysis of the reverse current transients at different temperatures. Finally, we employed an analytical method to determine the density and the energy distribution of the traps from SCLC current-voltage characteristics.
\end{abstract}

(c) 2014 Elsevier B.V. All rights reserved.

\section{Introduction}

Thanks to their high atomic number and wide band gap, cadmium telluride (CdTe) and cadmium zinc telluride (CdZnTe) have been widely proposed and used for room temperature X-ray and gamma ray detectors [1-5]. To enhance the charge collection, CdTe detectors are typically developed with blocking contacts (i.e. working as diodes), with indium (In) as the anode electrode on a p-type CdTe and platinum (Pt) as the cathode [2,3,6]. Blocking contacts are characterized by lower leakage currents than an ohmic configuration $(\mathrm{Pt} / \mathrm{CdTe} / \mathrm{Pt})$, allowing high bias voltage operation and accordingly better spectroscopic performance. Recently, new CdTe diode detectors are fabricated by using aluminum ( $\mathrm{Al}$ ) as blocking contact [7-11], allowing pixelization of the anode (critical issue of In contacts) and then the development of unipolar devices [3]. As widely reported in the literature [1,3], pixelization of the anode gives CdTe detectors unipolar properties (signals are mainly influenced by the electrons), very helpful to minimize the effects of the poor transport properties of the holes (critical issue of CdTe) in detector signals. Therefore, the high bias voltage operation and the fine segmentation of the electrodes of $\mathrm{Al} / \mathrm{CdTe}$ pixel detectors make them very attractive for high-resolution spectroscopic imaging, recently proposed in astrophysics, diagnostic medicine,

\footnotetext{
* Corresponding author. Tel.: + 39091 23899081; fax: + 3909123860815.

E-mail address: leonardo.abbene@unipa.it (L. Abbene).
}

industrial imaging, and security screening. Time instability under bias voltage (generally termed as polarization) is the major drawback of CdTe diode detectors, as well documented in the literature [12-14].

From the electrical point of view, polarization produces strong changes of the reverse current with time (both monotonic and non-monotonic current transients), while losses in detection efficiency, energy resolution and a progressive shift of the photopeaks toward lower energies are clearly visible in the measured $\mathrm{X}$-ray spectra. Degradations occur more rapidly at high temperatures, at low bias voltages and for thick detectors, as widely shown in several works [10,12-17]. Several solutions have been proposed to suppress polarization: high bias voltage operation, low temperature, low detector thickness, and switching off the bias voltage at regular time intervals. Polarization is mainly related to the accumulation of negative charge on deep acceptor levels during the application of the bias voltage (charge accumulation model, CAM) $[11,15,16]$.

As confirmed in [16], the origin of the deep centers responsible for the polarization in CdTe detectors is controversial. In this context, for example, the near mid-gap trap level $(\approx 0.6 \mathrm{eV})$ frequently found in CdTe crystals is attributed to the Cd vacancies, as in $[11,15]$. Conversely, in [17] the authors obtained the value of $0.47 \mathrm{eV}$ for this type of defect by using the electron paramagnetic resonance technique.

In our previous works $[7,8]$, the CAM model was confirmed on planar Schottky Al/p-CdTe/Pt detectors through both electrical and 
spectroscopic measurements, showing the dynamic of the polarization even for times $<1 \mathrm{~s}$. In this paper, we present the results of the electrical characterization of a $(4 \times 4)$ pixelated Schottky Al/p$\mathrm{CdTe} / \mathrm{Pt}$ detector. To better understand the basic properties and the carrier transport mechanisms of these devices, we investigated the current-voltage characteristics and the reverse current transients at different temperatures, comparing the results with those obtained for the planar Al/p-CdTe/Pt detectors [7,8]. Moreover, through the space charge limited current (SCLC) analysis in reverse bias, we also estimated the concentration and energy position of deep acceptor levels.

\section{Detectors and Experimental Procedures}

Electrical investigations were performed on a CdTe Schottky detector with pixelated anode $(\mathrm{Al} / \mathrm{Au} / \mathrm{Ti} /)$ and planar platinum $(\mathrm{Pt})$ cathode, deposited on a CdTe crystal $\left(4 \times 4 \times 2 \mathrm{~mm}^{3}\right)$ manufactured by Acrorad (Japan). The anode surface consists of 16 pixels arranged in a $4 \times 4$ array. The array is characterized by a pixel pitch of $1 \mathrm{~mm}$ in both directions: $0.6 \mathrm{~mm}$ is the pixel size with a gap of $0.4 \mathrm{~mm}$. For comparison, we also used a planar $\mathrm{Al} / \mathrm{p}-\mathrm{CdTe} / \mathrm{Pt}$ detector ( $2 \mathrm{~mm}$ thick with a guard ring around the anode) described in our previous works [7,8].

The detector was enclosed in a shielded box placed on a Peltier thermal stage with temperature control within $0.1^{\circ} \mathrm{C}$, and filled with nitrogen gas to prevent condensation. A custom interface was developed and used to separately measure the current of each pixel. This interface allows to connect each investigated pixel to the Keithley 236 Source Measure Unit (SMU) configured as electrometer, while the other pixels are fixed at zero voltage $[18,19]$. The SMU Keithley 2410 supplies the bias voltage to the cathode. The 2410 and 236 SMUs are synchronized with the possibility to vary the delay time $t_{\text {delay }}$ between the setting of the source voltage and the starting of the current measuring phase [7]. The current transients at fixed reverse bias voltage $\left(V_{R}=500 \mathrm{~V}\right)$ were performed by using the same setup with a sampling time of $\approx 1 \mathrm{~s}$. Before each waveform acquisition, the detector was biased at zero volt for a time long enough to avoid residual polarization effects. Computer control was used to perform all the measurements and the data acquisition. All measurements were performed under dark condition.

\section{Results and discussion}

\subsection{Current-voltage characteristics}

Fig. 1 shows the reverse current map of the 16 pixels of the CdTe pixel detector $\left(T=15^{\circ} \mathrm{C}, V_{\mathrm{R}}=1000 \mathrm{~V}\right.$, and $\left.t_{\text {delay }}=100 \mathrm{~ms}\right)$. Peripheral pixels are characterized by higher currents than the central ones, probably due to the absence of the guard ring which reduces the leakage currents. Moreover, we observed that the values of the pixel current are one order of magnitude higher than those of the CdTe planar detectors $(<1 \mathrm{nA})$, which are realized with a guard ring placed around the anode [7,20]. This result indicates the importance of the guard ring in the development of low noise detectors.

The current-voltage $(I-V)$ curves of the pixel no. 6 , measured at different temperatures $\left(15-45^{\circ} \mathrm{C}\right)$, are shown in Fig. 2, pointing out the rectifying property of the $\mathrm{Al} / \mathrm{CdTe}$ contact.

Linearity in the log-log plot of forward $I_{\mathrm{F}}-V_{\mathrm{F}}$ curves at $T=25^{\circ} \mathrm{C}$, shown in Fig. 3, is a strong evidence that thermionic emission, typical of metal/semiconductor contacts [21], does not occur at the forward bias. The power law $I_{\mathrm{F}} \alpha V_{\mathrm{F}}^{m}$ curves are characterized by two different regimes: at low voltages, i.e. for $V_{\mathrm{F}}<30 \mathrm{~V}, m<2$, and at

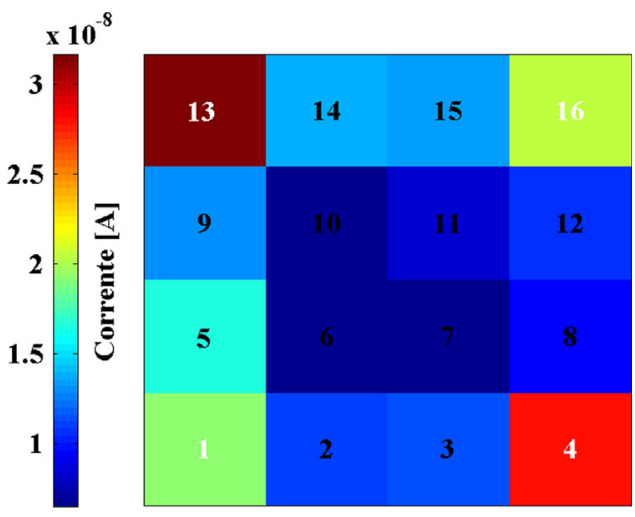

Fig. 1. Reverse current map of the 16 pixels of the CdTe pixel detector $\left(T=15^{\circ} \mathrm{C}\right.$, $\left.V_{R}=1000 \mathrm{~V}\right)$.

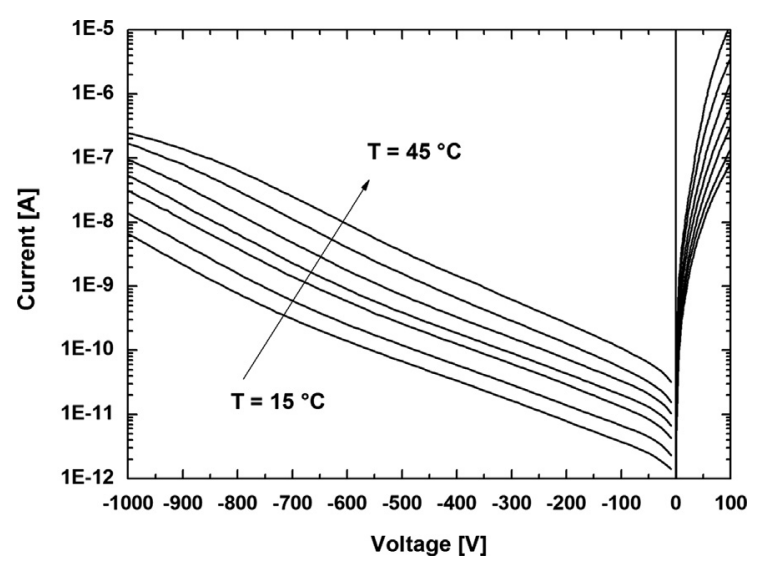

Fig. 2. $I-V$ curves of the pixel no. 6 measured at different temperatures.

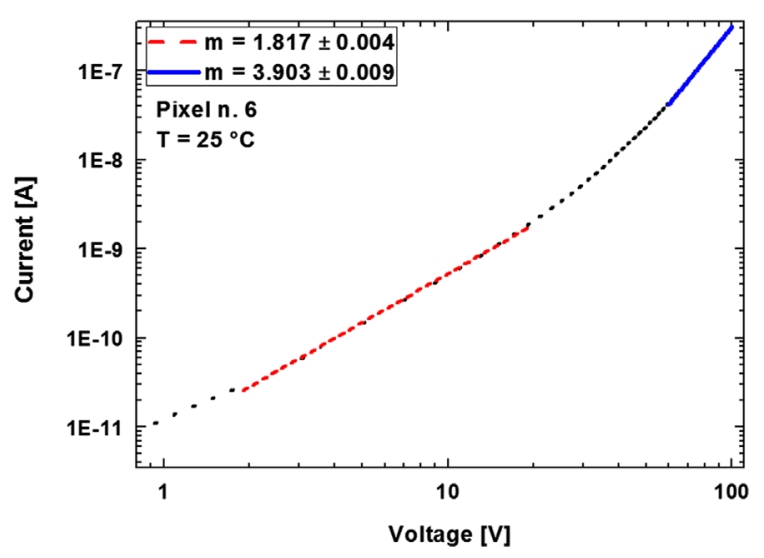

Fig. 3. Forward $I-V$ curve of the pixel no. 6 at $T=25^{\circ} \mathrm{C}$. The lines are the curve fitting functions $\left(I_{F} \alpha V_{F}^{m}\right)$ in the two bias voltage ranges.

higher voltages $m>2$. In the region where $m>2$, the current is governed by the space-charge-limited current (SCLC) mechanism, which is typical for semi-insulating materials [22,23]. According to this model, the deviation of $m$ from 2 is due to the presence of deep traps in the semi-insulating material. Therefore, under forward bias, deep traps play a key role in the transport mechanism.

Concerning the reverse currents, shown in Fig. 4, we observed an exponential trend at low voltage, i.e. at $V_{R}<200 \mathrm{~V}$, followed by a quick raising of the current values (i.e. $I_{R} \alpha V_{R}^{m}$ with $m>2$ ), also highlighting under reverse bias the presence of the SCLC transport mechanism. Moreover, the exponent $m$, calculated as $d \ln (I) / d \ln$ 


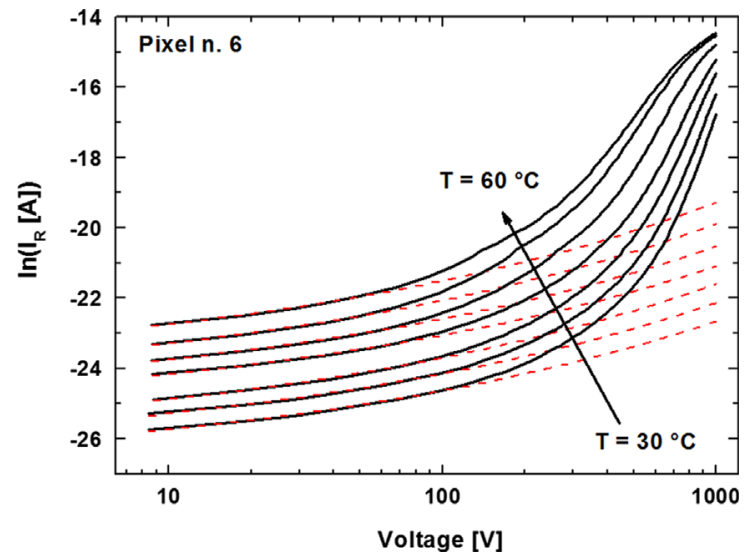

Fig. 4. Logarithm of the reverse current versus the bias voltage in the $30-60{ }^{\circ} \mathrm{C}$ temperature range for pixel no. 6 . The dashed lines are the curve fitting functions, with the logarithm of Eq. (6).

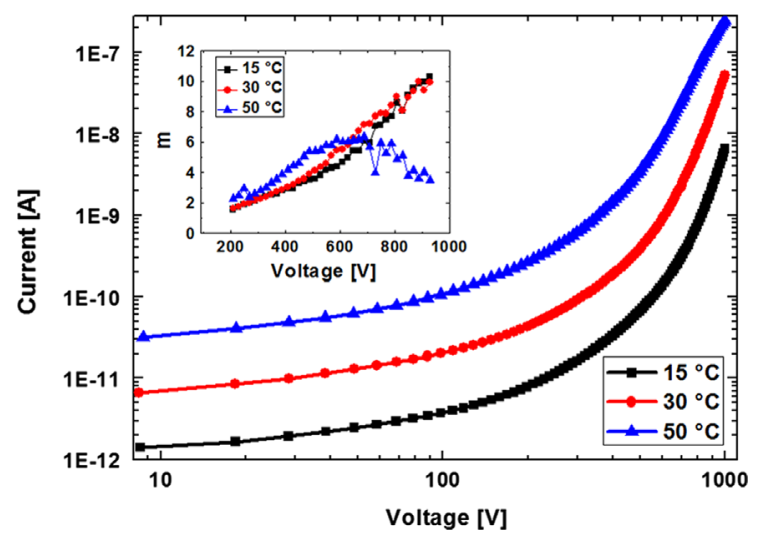

Fig. 5. Reverse $I-V$ and $m-V$ curves of the pixel no. 6 at different temperatures. The slope $m$ is given by $d \ln \left(I_{R}\right) / d \ln \left(V_{R}\right)$.

$(V)$, depends on the bias voltage and the temperature as shown in Fig. 5, where the $m-V$ curves at three different temperatures are reported. At low temperature, $m$ increases monotonically with the voltage. When $m$ exceeds the value of 2 , the deep traps begin to fill and accordingly the current varies more quickly with the bias voltage compared to the case of trap free material $(m=2)$. At higher temperatures, the $m-V$ curves show a peak due to the traps-filled-limit (TFL) which causes the decrease of the $m$ value at higher voltages. For single traps located at one energy level, the $I-V$ curve is characterized by nearly vertical rise in current at $V=V_{\mathrm{TFL}}$, where $V_{\mathrm{TFL}}$ is the voltage required to fill the traps. For $V>V_{\mathrm{TFL}}$ the exponent $m$ again takes the value 2 owing to SCLC in trap-free materials. Therefore, the continuous trend of the $m-V$ curves of the investigated pixels reveals continuous energy distributions of the traps in the mid-gap.

Concerning the time stability of the device, we observed a negligible time dependence of $I-V$ characteristics within time intervals less than $1 \mathrm{~s}$. In particular, the time dependence of the $I-V$ curves is not so marked when $t_{\text {delay }}$ varies in the range $40 \mathrm{~ms}^{-1} \mathrm{~s}$. Conversely, a strong time dependence was observed in the measured $I-V$ curves of planar $\mathrm{Al} / \mathrm{p}-\mathrm{CdTe} / \mathrm{Pt}$ detectors $[7,8]$.

Hence, we can neglect the time dependence of the $I-V$ characteristics and use the analysis of these curves for the determination of the charge transport mechanisms of the pixels.

\subsection{Al/CdTe barrier height}

In this section, we demonstrate that the reverse currents at low voltages $\left(V_{\mathrm{R}}<200 \mathrm{~V}\right)$ follow the voltage dependence predicted by the thermionic emission model. The reverse current of Schottky diode due to thermionic emission is given by [24]

$I_{\mathrm{R}}=A^{* *} T^{2} \exp \left(-\frac{q \varphi_{\mathrm{B} 0}}{k_{\mathrm{B}} T}\right) \exp \left(\frac{q \Delta \varphi_{\mathrm{b}}}{k_{\mathrm{B}} T}\right)$

where $A^{* *}$ is the product of the effective area and the effective Richardson constant $\left(A^{*}\right), k_{\mathrm{B}}$ is the Boltzamnn's constant, $q$ is the electron charge, $\phi_{\mathrm{B} 0}$ is the Schottky barrier height, and $\Delta \phi_{\mathrm{b}}$ is the Schottky barrier lowering. We used model (1) to extract the value of the barrier height $\phi_{\mathrm{B}}$ of the $\mathrm{Al} / \mathrm{CdTe}$ junction. For the planar detectors investigated in [7] Eq. (1) holds only for small voltages (near zero voltage) and so the contact resistance has been used to extract the value of the barrier height $\phi_{\mathrm{B} 0}$.

Generally, $\Delta \phi_{\mathrm{b}}$ is characterized by two terms $[11,21,24]$ :

$\Delta \varphi_{\mathrm{b}}=\left(\frac{q E}{4 \pi \varepsilon_{\mathrm{s}}}\right)^{1 / 2}+\alpha E$

where $\varepsilon_{\mathrm{s}}=10.2 \varepsilon_{0}$ is the CdTe dielectric constant [25]. The first term is due to the image force lowering caused by the electrical field at the anode $E$ and the second to the presence of a thin interfacial dipole layer between metal and semiconductor, where $\alpha$ is a constant [24]. Generally, the dipole lowering prevails at high values of the electrical field. Therefore, at low voltages we can assume that the image force lowering dominates.

If single dominant deep trap acceptor level with density $N_{\mathrm{T}}$ it is assumed, when a reverse bias voltage is applied at time $t=0$, the concentration of the ionized deep acceptors $N^{-}$will change as $N^{-}(t)=N_{\mathrm{T}}\left(1-e^{-t / \tau}\right)$, where $\tau$ is the hole detrapping time. Hence, the following expression of the time evolution of the electric field at anode $[11,26]$ holds:

$E(t)=\frac{q N_{\mathrm{T}}\left(1-e^{-t / \tau}\right)}{2 \varepsilon_{\mathrm{S}}} L+\frac{V_{\mathrm{R}}}{L}$,

where $L$ is the detector thickness.

As clearly described in [26], generally the conventional polarization model used in $[8,11,15,27-29]$ assumes that the bias voltage develops across all the detector thickness $L$. In [26], the authors suppose that almost all the bias voltage develops across the depletion width $W$. In our case, as we will show in the transient measurements, the formation of the dead layer occurs within the first few seconds after the bias of the detector.

Therefore, we must replace in Eq. (3) the detector thickness $L$ with the depletion width $W$ expressed as [16]

$W(t)=\sqrt{\frac{2 \varepsilon_{\mathrm{S}} V_{\mathrm{R}}}{q N_{\mathrm{T}}\left(1-e^{-t / \tau}\right)}} \leq L$

Thus, we can write the expression of the electrical at the anode at the generic time $t$ after biasing:

$E(t)=\sqrt{\frac{2 q N_{\mathrm{T}}\left(1-e^{-t / \tau}\right) V_{\mathrm{R}}}{\varepsilon_{\mathrm{S}}}}$

By using Eq. (5), we obtain the following expression of the reverse current as a function of the reverse voltage:

$I_{\mathrm{R}}=I_{1} \exp \left(\frac{q^{3 / 2}}{k_{\mathrm{B}} T \sqrt{4 \pi \varepsilon_{\mathrm{s}}}} \sqrt[4]{\left.\frac{2 q N_{\mathrm{T}}\left(1-e^{-t / \tau}\right) V_{\mathrm{R}}}{\varepsilon_{\mathrm{S}}}\right)}\right.$

where

$I_{1}=A^{* *} T^{2} \exp \left(-\frac{q \varphi_{\mathrm{B} 0}}{k_{\mathrm{B}} T}\right)$

We noted that expression (6) is valid only for $t>0$, i.e. when the process of ionization of the defects has already produced the dead layer and that the time taken for the acquisition of one $I-V$ curve is small compared to the time constant $\tau$. Hence, during the 
$I-V$ measurement the time-dependent term of Eq. (6) is kept almost constant.

In Fig. 4 are shown the measured curves of the reverse current of the central pixel no. 6 fitted with the logarithm of Eq. (6). We extracted the parameter $I_{1}$ from the intercept value of the best fit lines of the $\ln \left(I_{R}\right)-V_{R}$ curves shown in Fig. 4. The value of barrier height $\phi_{\mathrm{B}}$ can be extracted from the slope of the Arrhenius plot ln $\left(I_{1} / T^{2}\right)$ versus $1 / k_{\mathrm{B}} T$ (see Fig. 6 ).

The estimated values of the $\mathrm{Al} / \mathrm{CdTe}$ barrier height for all pixels are shown in Fig. 7 and the mean value over the pixels is $0.73 \pm 0.01 \mathrm{eV}$. This value is in good agreement with the barrier value obtained in planar detectors with the resistance contact method [7]. With similar detectors [30], the authors found values of the $\mathrm{Al} / \mathrm{CdTe}$ barrier height in the range $0.2-0.6 \mathrm{eV}$ as a function of the CdTe surface He plasma treatment.

\subsection{Transients of the reverse current}

The time evolution of the reverse current was measured within a time window of about $1200 \mathrm{~s}$ at reverse bias $V_{R}=500 \mathrm{~V}$ in the temperature range $24-44{ }^{\circ} \mathrm{C}$. Fig. 8 shows the transient of the reverse current at $T=24^{\circ} \mathrm{C}$ for different pixels. Although the central pixels are characterized by lower current values than the peripheral ones, all transient curves show similar behaviors. Hence the current offset between central and peripheral pixels is probably due to surface leakage current.

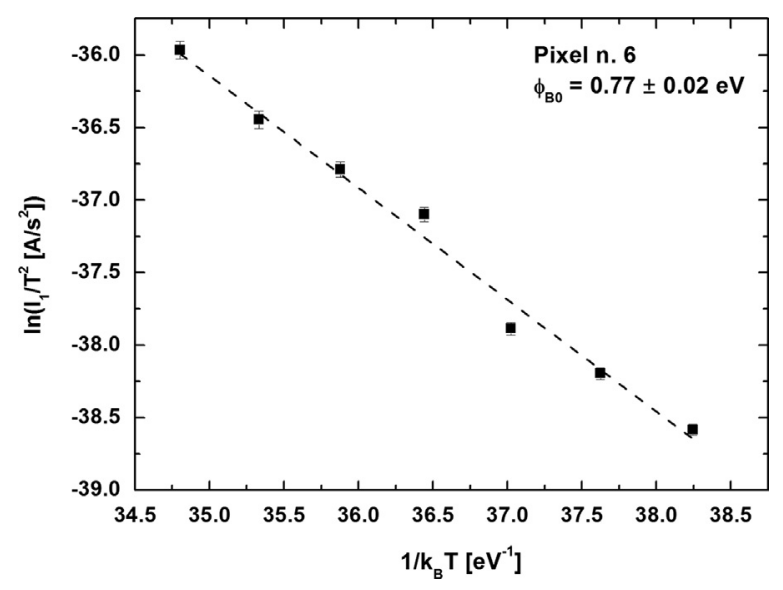

Fig. 6. Arrhenius plot of the current $I_{1}$ for the pixel no. 6 of the CdTe pixel detector.

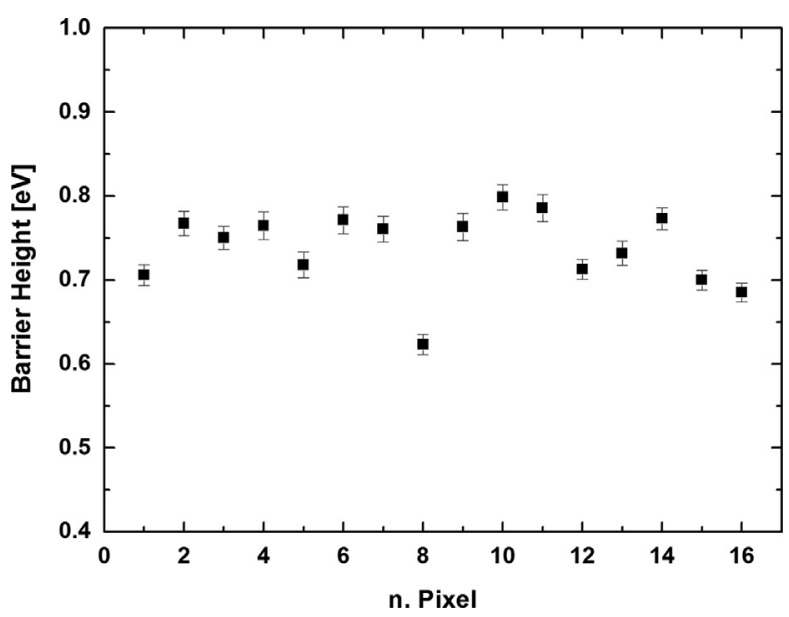

Fig. 7. Al/CdTe barrier heights of the 16 pixels of the detector.

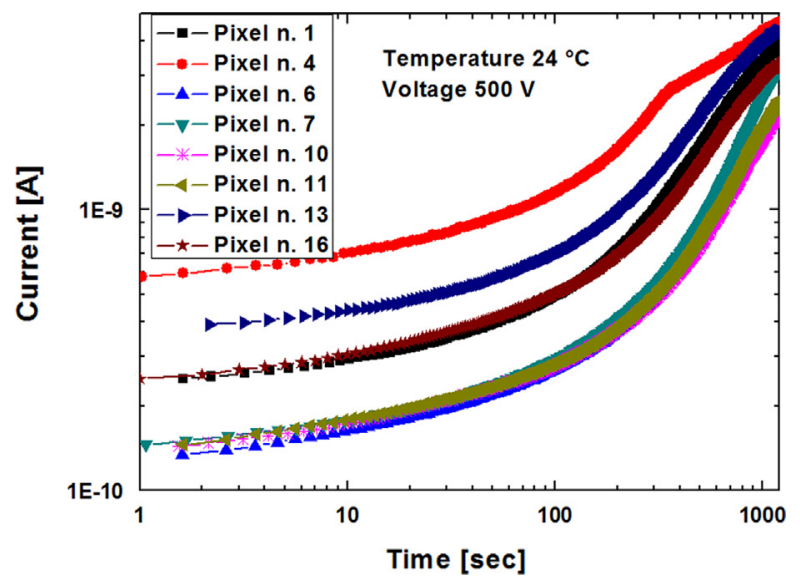

Fig. 8. (color online) Transient current curves under reverse bias $V_{\mathrm{R}}=500 \mathrm{~V}$, at $T=24{ }^{\circ} \mathrm{C}$ for different pixels.

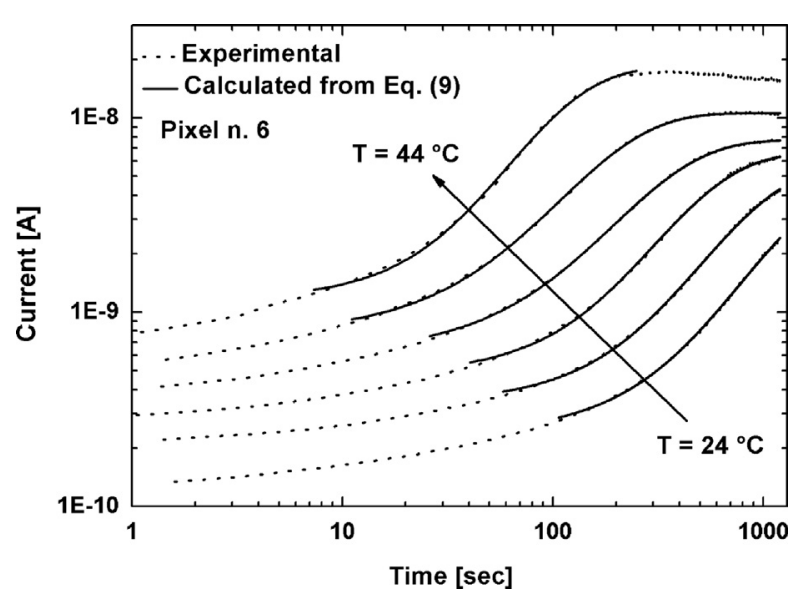

Fig. 9. Transient current curves under reverse bias $V_{R}=500 \mathrm{~V}$ measured in the temperature range $24-44^{\circ} \mathrm{C}$ for pixel no. 6 (dashed lines). The continuous lines are the fitting functions with Eq. (9).

Fig. 9 shows the transients measured in the temperature range $24-44{ }^{\circ} \mathrm{C}$ for the pixel no. 6 . The reverse current increases with time and tends to reach a plateau. At $T=44{ }^{\circ} \mathrm{C}$, the current transient exhibits the plateau after about $200 \mathrm{~s}$, time that decreases with the temperature increasing. This plateau is explained by the saturation of the electric field at the anode, which causes the decrease of the effective potential difference between anode and cathode [31]. According to the CAM model, the increasing of the reverse current at fixed bias is due to the holes emitted from trap levels, which generate a negative space charge near the anode and the creation of the dead layer near the cathode.

During this process, the carrier transport mechanism is governed by the ionization process of deep levels. Therefore, the increasing of the current is caused by the increasing of the electric field at the anode and by its decreasing at the cathode. The initial decreasing of the current, clearly visible in our previous transient measurements for planar detectors [7,8], was not observed in the investigated time window. This initial current decreasing is due to electron emission from the cathode occurring before the dead layer is formed. Moreover, the current plateau of pixels is reached in a time shorter than the planar one, as shown in Fig. 10. For the planar detector, the current reaches the plateau after about $10^{4} \mathrm{~s}$, while after about $2 \times 10^{2} \mathrm{~s}$ for the pixel at $T=40^{\circ} \mathrm{C}$. Therefore, the dynamic of the polarization of the pixel detector is within a time window shorter than the planar one. The shorter time scale of the polarization dynamic in the pixel detector justifies the absence of 


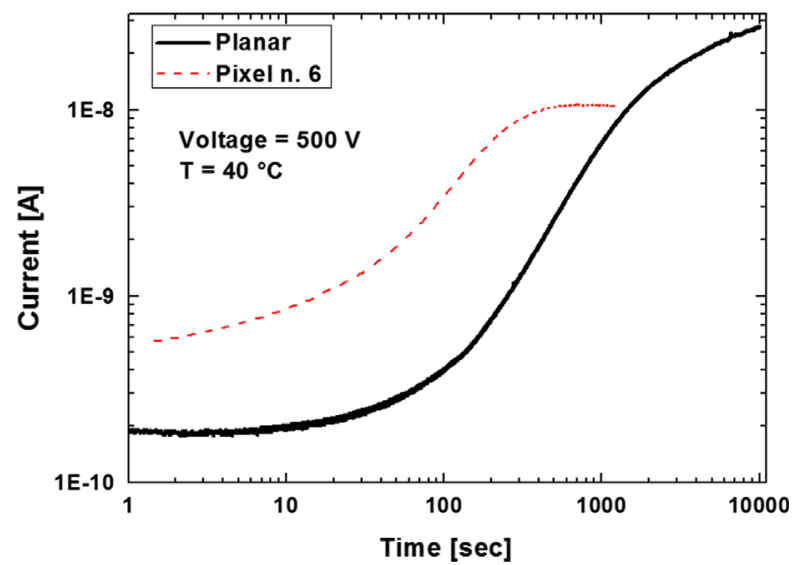

Fig. 10. Transient measurements under reverse bias $\left(V_{R}=500 \mathrm{~V} ; T=40{ }^{\circ} \mathrm{C}\right)$ of both the planar and the pixel detector.

the initial decreasing current, which occurs in a time scale $(<1 \mathrm{~ms}$ ) too short if compared with the sampling time of the used acquisition system. This difference also explains the weak time dependence of the curves for the pixel detector.

The shorter time scale of the polarization dynamic can be qualitatively explained as follows: the charge exchange with the deep traps, which is the main cause of polarization, can be modeled with the capacitance of the traps charged by the current flowing into the detector. The pixel detector has a lower resistance, due to the higher currents, than the planar detector (Fig. 10), and it has a lower capacitance because the area of the detector array pixel is about $1 / 10$ of the planar one. Hence, the time constant of the pixel detector is a few orders of magnitude smaller that of the planar detector and this explains the shorter time scale of the polarization dynamic.

By assuming a single dominant deep acceptor level, we can affirm that, when the current increases with time, the dead layer is already formed, and this justifies the replacement of the detector thickness $L$ with the depletion width $W$ in Eq. (3). This substitution is also correct when we used the $I-V$ curves to extract the barrier height. In fact, the current transient dynamic is faster than the planar detector ones and also at lower temperature the dead layer is formed in a time window shorter than $1 \mathrm{~s}$.

By analyzing the time evolution of the current at high reverse bias voltage, i.e. $V_{\mathrm{R}}>200 \mathrm{~V}$, we assumed that the barrier lowering at the interface $\mathrm{Al} / \mathrm{CdTe}$ is mainly due to the presence of a thin interfacial dipole layer between metal and semiconductor and so can be expressed with the second term of Eq. (2). Therefore, the relation between the time evolution of the current and the field at the anode can be expressed combining Eq. (2) and Eq. (1):

$I_{\mathrm{R}}(t)=I_{1} e^{[q \alpha E(t)] /\left(k_{\mathrm{B}} T\right)}$

Combining expressions (5) and (8), we obtained the model of the time dependence of reverse current used to fit experimental data expressed as

$I_{\mathrm{R}}(t)=I_{1} \exp (b \sqrt{1-\exp (-t / \tau)})+c$

where $b=\left(\alpha q /\left(k_{\mathrm{B}} T\right)\right) \sqrt{2 q N_{\mathrm{T}} V_{\mathrm{R}} / \varepsilon_{\mathrm{S}}}$ and $c$ is the current value at the instant time where Eq. (9) begins to be valid, that is after the formation of the dead layer. In Fig. 9, the measured curves of the reverse current fitted with relation (9) are reported. We noted that the range of the fitting function increases at high temperature. In fact, at high temperature, the formation of dead layer occurs earlier than that at low temperature.

From the fitting of the transient curves at different temperatures we estimated the time constant $\tau$. In Fig. 11 is shown the Arrhenius plot of $\tau$ for the pixel no. 6, obtaining the activation

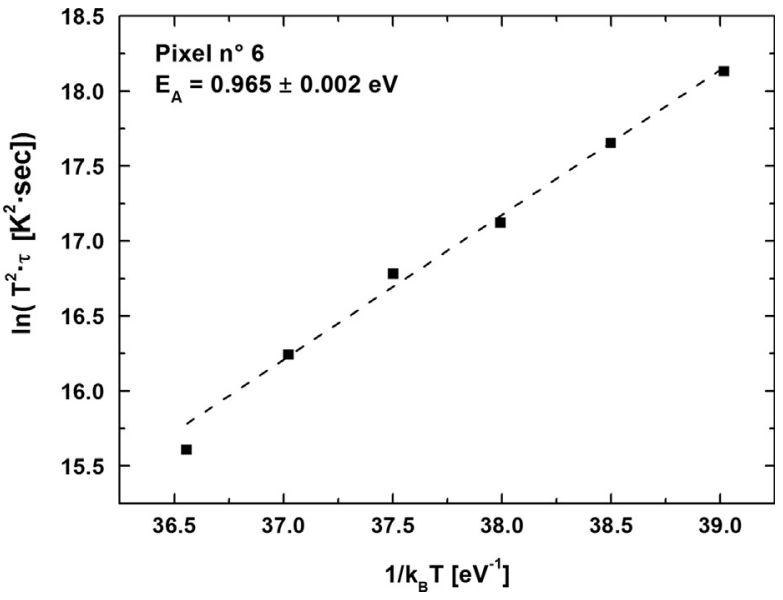

Fig. 11. Arrhenius plot of the time constant $\tau$ obtained from the model (9) of the reverse current transient at $V_{R}=500 \mathrm{~V}$.

energy $E_{\mathrm{A}}=0.96 \mathrm{eV}$ (the mean value for all pixels is $0.85 \pm$ $0.02 \mathrm{eV}$ ). The activation energy values are slightly higher than the planar detector ones $[7,8]$. This difference is due to the approximation used in our previous works, where the fitting of the current transient has been performed in a time interval where $t<\tau$, due to the slower polarization dynamic of the planar detector. For this reason, for planar detectors $[7,8]$ we used the expression of the electrical field given by Eq. (3) instead of Eq. (5). Thus, in this case the activation energy of the time $\tau$ constant multiplied by a factor dependent on the concentration of the ionized acceptors has been estimated. Deep levels with energies in the range $0.6-0.8 \mathrm{eV}$ in $\mathrm{CdTe}$ were found by several groups and were evaluated by different methods [11,28,29,31-34]. These energy levels are often attributed to acceptor levels responsible for polarization which might depend on the thermal treatment during the crystal growth [35].

\subsection{SCLC analysis}

We investigated the reverse $I-V$ curves, where the SCLC mechanism dominates, by using the differential analysis of the SCLC developed by Nešpurek and Sworakowski [35,36], which allows to determine the energy distribution and the concentration of traps in the band gap without any a priori assumptions concerning their energy distribution. The boundary conditions required to solve the differential equations are: (i) the current is carried by only one type of charge (holes in our case); (ii) the band edge is chosen as reference point of the energy; (iii) the diffusion current is neglected; (iv) the density of free carriers can be described by the Boltzmann statistics and trapped carriers by the Fermi-Dirac statistics; (v) quasi-equilibrium condition is reached at any injection rate, and is characterized by a common quasiFermi level. Some of these boundary conditions are discussed below.

The weak time dependence of the reverse $I-V$ curves ensures that the electron emission from the cathode has lapsed and the detector current is dominated by holes emitted by traps $[7,8]$. Hence, in the reverse SCLC regime, only one type of charge dominates the transport mechanism. SCLC in reverse has also been observed in In/CdTe/Pt detectors by Cola [37]. In this case, the presence of SCLC in reverse is due to electrons injected at the cathode. This assertion is confirmed considering that with high reverse voltage ( $V_{R}=600 \mathrm{~V}$ in this case) the authors find a non-null electrical field at the cathode, which vanishes after a time of $300 \mathrm{~s}$ at $T=40{ }^{\circ} \mathrm{C}$. When the electrical field becomes null at the cathode, the current increases with time and is dominated by the holes 
through the generation mechanism in the space charge layer (i.e. $\left.I_{R} \alpha V_{R}^{1 / 2}\right)$. The absence of SCLC in In/CdTe/Pt detectors, when the current is dominated by holes emitted from ionized acceptors, is due to the higher In/CdTe barrier height $(1 \mathrm{eV})$ than the $\mathrm{Al} / \mathrm{CdTe}$ one $(0.7 \mathrm{eV})$. Hence, the hole injecting efficiency of the $\mathrm{Al} / \mathrm{CdTe}$ contact is higher than that of the In/CdTe contact and this causes the hole space charge formation at high reverse voltage in our pixel detectors. Whereas the better hole blocking nature of the In/ CdTe contact prevents the formation of the hole SCLC and also delays the electrical field decrease at the cathode. Therefore, in our case, when the reverse $I-V$ curves show the SCLC mechanism, the reverse current is dominated by holes emitted from traps. We did not use the Nešpurek and Sworakowski method to analyze the SCLC at forward bias, because it is not really clear if the current is carried out only by one type of charge and if diffusion mechanisms are present.

Using the Nešpurek and Sworakowski method, we assume that the holes are thermally excited from the localized traps to delocalized band states. This assumption allows to choose as the reference point for the energy scale the valence band edge (i.e. $E_{\mathrm{V}}=0$ ), with positive energy toward the mid-gap. At thermal equilibrium, the density of trapped holes $p_{t}\left(\mathrm{~cm}^{-3}\right)=$ $\int_{E} h(E) f\left(E-E_{\mathrm{F}}\right) d E$ and the density of free charges $p_{f}\left(\mathrm{~cm}^{-3}\right)=$ $N_{V} \exp \left(-E_{F} / k T\right)$ are characterized by a common quasi-Fermi level $E_{\mathrm{F}}$ and can be described by Fermi-Dirac and Boltzmann statistics. $N_{\mathrm{V}}=2\left(2 \pi m_{\mathrm{h}} k T / h^{2}\right)^{3 / 2} \mathrm{~cm}^{-3}$ is the effective density of states (DOS) in the valence band, $m_{\mathrm{h}}$ is hole effective mass equal to $0.35 m_{\mathrm{e}}$ [38], $f$ is the Fermi-Dirac function, and $h(E)$ is the energy distribution of the density of allowed states.

We can suppose the quasi-equilibrium condition because the I$V$ curves are acquired in a time window where polarization effects are not so marked.

By using the zero temperature approximation, so that the quasi-Fermi level represents the demarcation between empty and filled traps, the trap distribution is given by [36]

$h\left(E_{\mathrm{F}}\right)=\left(\frac{\varepsilon_{s} x_{1} x_{2}}{q L^{2} k T}\right)\left(\frac{V}{m-1}\right)\left(\frac{1+C}{1+B}\right)$

where $x_{1}, x_{2}, B$, and $C$ are coefficient functions of $m$ [36]. Thus, neglecting the diffusion current, the current density at the anode is $j=e \mu p_{\mathrm{f}} E$.

Using the relation between the potential and the electric field $E=x_{1} V_{\mathrm{R}} / L$ the position of the quasi-Fermi level can be expressed as

$E_{\mathrm{F}}=k T \ln \left(\frac{e \mu_{p} N_{V} x_{1}}{L}\right)+k T \ln \left(\frac{V_{\mathrm{R}}}{I_{\mathrm{R}}}\right)$

where $\mu_{\mathrm{p}}=57\left(e^{252 / T}-1\right) \mathrm{cm}^{2} / V \mathrm{~s}$ is the mobility of the charge carriers [39]. We investigate the effect of the temperature in this analysis of DOS profile of the dominant traps, obtained from $I-V$ curves. The energy value of Fermi level extracted with Eq. (12) is sensitive to the parameters $\mu_{\mathrm{p}}, N_{\mathrm{V}}$, and $m_{\mathrm{h}}$. A variation of $\sim 10 \%$ in these parameters causes a variation $\sim 0.01 \mathrm{eV}$ in the value of the quasi-Fermi level.

Fig. 12 shows the concentration of the traps as a function of the quasi-Fermi level position for the pixel no. 6 at three temperatures $\left(15,30\right.$, and $\left.50^{\circ} \mathrm{C}\right)$. The quasi-Fermi level position varies in the range $0.8-0.9 \mathrm{eV}$ and the concentration of traps is approximately of the order of $10^{11}-10^{12} \mathrm{~cm}^{-3}$, which is in agreement with the values reported in literature for planar CdTe detectors and obtained with several methods $[35,36]$. Moreover, the $h\left(E_{\mathrm{F}}\right)$ peak at $E_{\mathrm{F}} \approx 0.88 \mathrm{eV}$ at $T=15{ }^{\circ} \mathrm{C}$ decreases when the temperature increases until it disappears at $T=50{ }^{\circ} \mathrm{C}$. This effect is due to the filling of the traps with the temperature increasing, which limits the exchange between the injected holes and the traps. Moreover, when the temperature increases the quasi-Fermi level intercepts

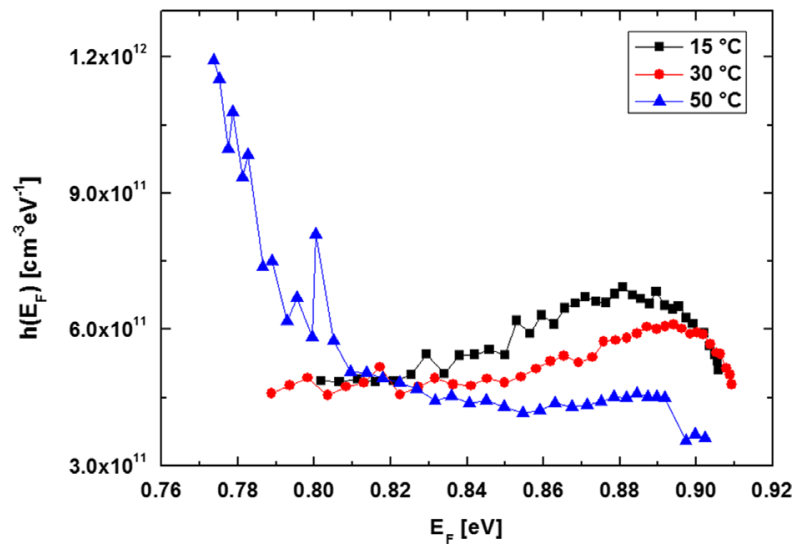

Fig. 12. Density of states of trap distribution obtained from the analysis of reverse $I-V$ curves at different temperatures of pixel no. 6 with Eqs. (10) and (11).

energy levels close to the valence band where $h\left(E_{\mathrm{F}}\right)$ tends to increase, probably due to the presence of defects located at shallower levels. This method allows investigations on a limited energy range $\left(\Delta E_{\mathrm{F}} \approx 0.2 \mathrm{eV}\right.$ ), which does not include the energy levels in the range $0.6-0.8 \mathrm{eV}$, where the traps, found with the transient current analysis, are located. Conversely, SCLC analysis gives indication of the concentration of the majority carrier traps in the investigated samples. In literature, SCLC analyses based on this approach were carried out in different semi-insulating materials. The concentration of the electrically active deep traps is generally obtained by several characterization methods, e.g. Photo Induced Current Transient Spectroscopy (PICTS), SPS (Surface Photovoltage Spectroscopy), Thermally Stimulated Current (TSC), and Thermally Stimulated Luminescence (TSL). As shown by Fraboni et al. [40,41], the activation energies of the dominating traps obtained in high resistivity CdTe with SCLC analysis is in agreement with the results obtained by different spectroscopic characterization methods (PICTS, SPS).

\section{Conclusions}

The electrical characterization of $\mathrm{Al} / \mathrm{p}-\mathrm{CdTe} / \mathrm{Pt}$ pixel detector was presented. Measurements of current voltage characteristics and transient characterization at different temperatures were performed to better understand the charge transport mechanisms of the CdTe Schottky detectors and the bias induced polarization. Of course, deep levels play a key role in the charge transport mechanism, with the SCLC mechanism that dominates under forward bias and at high reverse voltages. We estimated the Schottky barrier height of $\mathrm{Al} / \mathrm{CdTe}$ contact by using the thermionic-field emission model which only dominates at low reverse voltage $\left(V_{R}<200 \mathrm{~V}\right)$, obtaining a value of about $0.73 \mathrm{eV}$, in good agreement with literature. The detector showed a weak time dependence of $I-V$ curves within time intervals less than $1 \mathrm{~s}$. This result, contrary to the strong time dependence of curves in planar detectors (investigated in our previous works $[7,8]$ ), is due to the shorter time scale of the polarization dynamic than the planar one. The analysis of the reverse current transient performed at different temperatures allowed us to extract the activation energy of the deep levels (charge accumulation model). The activation energy $(0.73-0.96 \mathrm{eV})$ values are comparable with levels generally responsible of the polarization phenomenon. Finally, we employed a numerical method based on SCLC for the evaluation of the density distribution of traps. The SCLC differential method, performed only in reverse bias (where we demonstrated that the 
current is carried out only by holes), gave a deep acceptor level concentration of $10^{11}-10^{12} \mathrm{~cm}^{-3}$ in the range $0.8-0.9 \mathrm{eV}$.

\section{Acknowledgment}

This work was supported in part by the Italian Ministry for Education, University and Research (MIUR) under PRIN Project no. 2012WM9MEP and in part by the University of Palermo under FFR Project no. 2012-ATE-0153.

The authors would like to acknowledge the technical staff of the laboratory, Mr. Marcello Mirabello and Mr. Michele Quartararo for their technical assistance.

\section{References}

[1] A. Owens, A. Peacock, Nuclear Instruments and Methods in Physics Research Section A 531 (2004) 18.

[2] T. Takahashi, S. Watanabe, IEEE Transactions on Nuclear Science NS48 (2001) 950.

[3] S. Del Sordo, et al., Sensors 9 (2009) 3491.

[4] L. Abbene, S. Del Sordo, F. Fauci, G. Gerardi, A. La Manna, G. Raso, A. Cola, E. Perillo, A. Raulo, V. Gostilo, S. Stumbo, Nuclear Instruments and Methods in Physics Research Section A 583 (2007) 324.

[5] N. Auricchio, et al., Journal of Applied Physics 110 (2011) 124502.

[6] L. Abbene, G. Gerardi, F. Principato, S. Del Sordo, G. Raso, Sensors 12 (2012) 8390.

[7] F. Principato, G. Gerardi, A.A. Turturici, L. Abbene, Journal of Applied Physics 112 (2012) 094506

[8] F. Principato, A.A. Turturici, M. Gallo, L. Abbene, Nuclear Instruments and Methods in Physics Research Section A 730 (2013) 141.

[9] L. Abbene, G. Gerardi, A.A. Turturici, S. Del Sordo, F. Principato, Nuclear Instruments and Methods in Physics Research Section A 730 (2013) 135.

[10] A. Meuris, O. Limousin, C. Blondel, Nuclear Instruments and Methods in Physics Research Section A 654 (2011) 293.

[11] H. Toyama, A. Higa, M. Yamazato, T. Maehama, R. Ohno, M. Toguchi, Japanese Journal of Applied Physics 45 (2006) 8842.

[12] P. Siffert, J. Berger, C. Scharager, A. Cornet, R. Stuck, R.O. Bell, H.B. Serreze, F.V. Wald, IEEE Transactions on Nuclear Science NS23 (1976) 159.

[13] H.L. Malm, M. Martini, IEEE Transactions on Nuclear Science NS21 (1974) 322.

[14] R. Bell, G. Entine, H. Serreze, Nuclear Instruments and Methods 117 (1974) 267.
[15] A. Cola, I. Farella, Applied Physics Letters 94 (2009) 102113.

[16] R. Grill, E. Belas, J. Franc, M. Bugar, S. Uxa, P. Moravec, P. Hoschl, IEEE Transactions on Nuclear Science NS58 (2011) 3172.

[17] P. Emanuelsson, P. Omling, B.K. Meyer, M. Wienecke, M. Schenk, Physica Review B 47 (1993) 15578.

[18] K. Yang, B. Cha, D. Heo, S. Jeon, Journal of Instrumentation 7 (2012) C12030.

[19] B.P.F. Dirks, C. Blondel, F. Daly, O. Gevin, O. Limousin, F. Lugiez, Nuclear Instruments and Methods in Physics Research Section A 567 (2006) 145.

[20] W.C. Barber, N. Malakhov, M.Q. Damron, N.E. Hartsough, D. Moraes, P. Weilhammer, E. Nygard, J.S. Iwanczyk, IEEE Nuclear Science Symposium Conference Record 4 (2007) 2414.

21] S.M. Sze, Physics of Semiconductor Devices, 2nd ed., J. Wiley \& Sons, 1981.

[22] A. Rose, Physical Review 97 (1955) 1538.

[23] M. Lampert, Reports on Progress in Physics 27 (1964) 329.

24] J.M. Andrews, M.P. Lepselter, Solid-State Electronics 13 (1970) 1011.

[25] A. Manabe, A. Mitsuishi, H. Yoshinaga, Japanese Journal of Applied Physics 6 (1967) 593.

[26] H. Elhadidy, V. Dedic, J. Franc, R. Grill, Journal of Physics D: Applied Physics 47 (2014) 055104

[27] K. Suzuki, T. Sawada, K. Imai, S. Seto, IEEE Transactions on Nuclear Science NS59 (2012) 1522

[28] N. Remoué, D. Barret, O. Godet, P. Mandrou, Nuclear Instruments and Methods in Physics Research Section A 618 (2010) 199.

[29] M. Ayoub, M. Hage-Ali, J. Koebel, A. Zumbiehl, F. Klotz, C. Rit, R. Regal, P. Fougeres, P. Siffert, IEEE Transactions on Nuclear Science NS50 (2003) 229.

[30] H. Toyama, M. Yamazato, A. Higa, T. Maehama, R. Ohno, M. Toguchi, Japanese Journal of Applied Physics 44 (2005) 6742.

[31] A. Cola, I. Farella, A.M. Mancini, A. Donati, IEEE Transactions on Nuclear Science NS54 (2007) 868.

[32] A. Cavallini, D. Cavalcoli, A. Castaldini, B. Fraboni, IEEE Transactions on Nuclear Science NS54 (2007) 1719.

[33] A. Castaldini, A. Cavallini, B. Fraboni, P. Fernandez., J. Piqueras, Journal of Applied Physics 83 (1998) 2121.

[34] A. Cavallini, B. Fraboni, W. Dusi, M. Zanarini, Journal of Applied Physics 94 (2003) 3135

[35] S. Nešpurek, J. Sworakowski, Physica Status Solidi A 41 (1977) 619.

[36] J. Sworakowski, S. Nešpurek, IEEE Transactions on Electrical Insulation 24 (1989) 223.

[37] A. Cola, I. Farella, Applied Physics Letters 102 (2013) 113502.

38] P. Sydorchuk, G. Khlyap, Crystal Research and Technology 36 (2001) 1027.

[39] D. Kranzer, Physica Status Solidi A 26 (1974) 11.

[40] B. Fraboni, D. Cavalcoli, A. Cavallini, P. Fochuk, Journal of Applied Physics 105 (2009) 073705.

[41] B. Fraboni, L. Pasquini, A. Castaldini, A. Cavallini, P. Siffert, Journal of Applied Physics 106 (2009) 093713. 